\title{
Moralisierungen in Gastfamilien für unbegleitete Geflüchtete
}

\section{Differenzbearbeitung und Beziehungsgestaltung zwischen Gast und Familie ${ }^{1}$}

\section{Ines Gottschalk}

Journal für Psychologie, 28(2), 56-77

https://doi.org/10.30820/0942-2285-2020-2-56

CC BY-NC-ND 3.0 DE

www.journal-fuer-psychologie.de

\section{Zusammenfassung}

Im Beitrag werden Gastfamilien für unbegleitete Geflüchtete als moralische Handlungsräume verstanden, an die unterschiedliche Orientierungen der Moralisierung, aber auch moralische Werte herangetragen werden. Es wird gezeigt, dass Gastfamilien verschiedene Funktionen einnehmen, wenn sie den Unbegleiteten als Gast oder Familienmitglied verstehen, und dass dadurch auch differente Orientierungen und Werthaltungen anders bearbeitet werden können. Als sich neuformierender symbolischer Handlungsraum obliegt es den Gastfamilienmitgliedern, ihre Orientierungen und Wertvorstellungen im Rahmen alltäglicher Interaktionen auszuhandeln. Entlang einer qualitativen Einzelfallstudie aus Deutschland wird gezeigt, wie Moralvorstellungen, aber auch die Rolle des Unbegleiteten und die damit einhergehenden Spielräume der Moralisierung situativ und fluide mit verschiedenen Akteuren ausgehandelt werden. Davon ausgehend werden allgemeine Überlegungen zu Beziehungsgestaltungen und Subjektumbildungen im Zusammenhang mit Moral(-isierungen) als kontingente Orientierungen und Praktiken hergeleitet.

Schlüsselwörter: Unbegleitete Geflüchtete, Gastfamilie, Interkulturalität, Moral, Beziehungsaushandlung, Identität, Qualitative Forschung

\section{Summary}

Moralizations in host families for unaccompanied refugees. Difference management and relationship formation between host and family

In this contribution, host families for unaccompanied refugees are understood as moral action spaces to which different orientations of moralization and moral values are transmitted. It is shown that host families can take on different functions, depending on whether they understand the unaccompanied person as a family member or as a guest, and can therefore 
deal differently with divergent orientations and values. As a newly forming symbolic space of action, it is the obligation of the host family members to negotiate their orientations and values in the context of everyday interactions. Using a qualitative case study conducted in Germany it is shown how moral concepts, but also the role of the unaccompanied and the related possibilities for moralization are negotiated with different actors in a fluid and situational way. Based on this, general reflections on the configuration of relationships and the reformation of subjects in connection with moral(-ization) as contingent orientations and practices are derived.

Keywords: Unaccompanied refugees, host family, interculturality, moral, relationship negotiation, identity, qualitative research

Allein im Jahr 2016 wurden 44.935 minderjährige geflüchtete Personen, die ohne erwachsene, sorgeberechtigte Begleitperson Deutschland erreichten, in Obhut genommen (Tangermann und Hoffmeyer-Zlotnik 2018). Die Aufnahme sogenannter unbegleiteter minderjähriger Geflüchteter in eine Gastfamilie ${ }^{2}$ wird vom KompetenzZentrum Pflegekinder e. V. (Betscher und Szylowicki 2016) als Möglichkeit verstanden, Unbegleiteten Geborgenheit und Schutz zu vermitteln und eine individuelle, bedürfnisorientierte Unterstützung bei der Integration zu gewährleisten.

Gastfamilien, hier im weitesten Sinne als familienähnlicher Verbund verstanden, die gemeinsam einen ohne Eltern nach Deutschland geflüchteten Jugendlichen begleiten, stehen im Zentrum des Beitrags. Die skizzierten Erwartungshaltungen an Gastfamilien verdeutlichen bereits, dass sich Gastfamilien als Handlungsräume verstehen lassen, denen sowohl Orientierungen der Beziehungsgestaltung als auch moralische Inhalte nahegelegt werden. Im ersten Abschnitt wird Gastfamilie daher als ein über kulturelle Wissensbestände vorgeprägter moralischer Raum im Sinne von Charles Taylor (2016 [1992]) vorgestellt, der im Spannungsfeld zwischen »Gast « und »Familie « konstituiert wird.

Im zweiten Abschnitt wird gezeigt, dass sich die Gastfamilie in diesem Spannungsfeld (neu) formieren muss und zu gemeinsamen Orientierungen der Moral(-isierung) im Rahmen von alltäglichen Aushandlungen (Bergmann 1999) findet. Einblick in die familiäre Lebenswelt und die Aushandlung von Moral(-isierungen) wird im dritten Abschnitt anhand von Sequenzen aus einem Interview mit der Gastmutter Birgit ${ }^{3}$ gege- $^{-}$ ben. Die erweiterte Familie, die sich um den Unbegleiteten Hanad bildet, repräsentiert eine vielleicht zunächst ungewöhnlich erscheinende Form gelebter Gastfamilie, zeigt jedoch besonders anschaulich typische Probleme in der Gastfamilie auf. An den Narrationen von Birgit wird exemplarisch verdeutlicht, wie Moralisierungen, aber auch moralische Inhalte, in Beziehungen als fluide, situative Inhalte und Praktiken von den Akteuren mit sich selbst und anderen ausgehandelt werden. In der abschließenden 
Betrachtung werden Überlegungen zum Zusammenhang von Beziehungsgestaltungen und Subjektumbildungen im Rahmen von Moral(-isierungen) in familialen, interkulturellen Beziehungen angestellt, die sich - so die Argumentation - auch auf andere Beziehungsformen übertragen lassen.

\section{Die Gastfamilie als moralischer (Handlungs-)Raum: Differenzbearbeitung zwischen Gast und Familie}

In diesem Beitrag werden Gastfamilien aus kulturpsychologischer Perspektive als symbolische Handlungsräume (Boesch 1991) verstanden. Demnach werden an Individuen über Semantiken, gesellschaftliche und politische Wertungen sowie institutionelle Normierungen kulturelle Wissensbestände herangetragen, an denen sich Individuen orientieren und die sie sich aneignen können. Das heißt, sie können ihre Handlungsziele, ihre Praktiken danach ausrichten und sich selbst sowie ihre Welt, und somit auch ihre Beziehungen, dazu in Bezug setzen. Nun bewegt sich das Subjekt als handelndes Individuum immer durch verschiedene bereits mehr oder weniger normierte Handlungsräume und befindet sich damit immer in bestimmten wert- sowie sinnbezogenen Bedeutungskontexten. Da dadurch immer auch nahegelegt wird, was vermeintlich richtig oder falsch, besser oder schlechter ist, lassen sich Handlungsräume zugleich als moralische Räume im Sinne von Taylor (2016 [1992]) verstehen (Straub 1994, 11).

Auch neu entstehende Beziehungen lassen sich im Boesch'schen (1991) Sinne als Handlungsräume verstehen. Individuen, die miteinander in Verbindung treten, deuten aufgrund eines unterschiedlichen biografischen und kulturellen Hintergrunds ihre Beziehung unterschiedlich. Um ihrem gemeinsamen, ko-konstruktiv geschaffenen Handlungsraum Bedeutung zuzuschreiben, greifen die Individuen auf sogenannte promoter signs zurück und deuten ihre Beziehung beispielsweise als Freundschafts-, Familien- oder Arbeitsbeziehung. Diese Deutungsangebote gehen entsprechend auch mit unterschiedlichen Werthaltungen, wie die Beziehungen ausgestaltet werden und welche Normen und Werte für die Subjekte handlungsleitend sein sollten, einher. Entsprechend ist auch die Beziehungsform der Gastfamilie ein moralischer (Handlungs-)Raum, welcher durch unterschiedliche kulturelle Wissensbestände und divergierender Bedeutungen zu einem polyvalenten Beziehungskonzept wird.

Die Personen müssen die Bedeutungen, die sie ihrer Beziehung und sich wechselseitig geben, und die Wertorientierungen, die damit einhergehen, miteinander aushandeln. Es findet also eine gemeinsame Differenzbearbeitung statt. Beziehungen werden jedoch zumeist nicht auf einer Metaebene ausgehandelt, sondern im alltäglichen Miteinander. Im Anschluss an Jörg Bergmann (1999) lässt sich hier von (Alltags-)Moralisierungen sprechen, die über sozial wertende Stellungnahmen und Urteile, die sich auf Hand- 
lungen oder Personen beziehen, und über Wörter, Blicke oder Gesten kommuniziert werden. Zum einen dienen sie dazu, einen gemeinsamen Werterahmen und Orientierung zu schaffen. Zum anderen können Moralisierungen zu emotionalen Verletzungen und zu einem Bruch der sozialen Beziehung führen.

Inwiefern und in welcher Form die Beziehungsdeutung selbst als auch damit einhergehende Wertvorstellungen überhaupt verhandelbar sind und in welche Richtung moralisiert wird, hängt davon ab, wie die Beziehungen in diesem Handlungs- und Kulturraum normiert und hierarchisch (vor-)strukturiert sind. Beziehungen sind damit auch Handlungssphären im Boesch'schen Sinne und werden definiert als »culturally defined context of meaning [which] tends to organize space, time, social relationships and personal action orientations in a particular way (Boesch 1991, 72). Dementsprechend prägt auch die Deutung als »Gastfamilie« die Differenzbearbeitung über Praktiken der Moralisierung, Sozialisation und Normierung auf eine spezifische Art und Weise.

Um den polyvalenten (Vor-)Strukturierungen des sozialen Feldes auf die Spur zu kommen, soll hier zunächst rekonstruiert werden, welche Zielvorstellungen, Orientierungen und Subjektpositionierungen an die Gastfamilie angelegt werden. Dazu wird die Semantik » Gastfamilie « und ihre Valenzen in den Blick genommen. Es wird argumentiert, dass über die Begrifflichkeiten » Gast « und »Familie « und ihre polyvalenten Bedeutungen unterschiedliche, sich zum Teil widersprechende Orientierungen der Beziehungsgestaltung und somit auch der Subjektpositionierung nahegelegt werden. Für die Aushandlung und Differenzbearbeitung bedeutet das somit auch, dass je nach Deutung bestimmte Formen und Richtungen von Moralisierungspraktiken dominieren. Dazu werden zunächst Spezifika und Zielvorstellungen von Familien im idealtypischen Sinne in den Blick genommen, bevor auf Besonderheiten des Unbegleiteten als » Gast « in solch einem Setting eingegangen wird. Um zu zeigen, wie sich die Orientierungen zwischen »Gast « und »Familie « auch in institutionellen Zielvorstellungen für Gastfamilien widerspiegeln, werden Leitziele aus einer Broschüre für werdende Gasteltern (Becker 2016) sowie einer Publikation des Kompetenz-Zentrums Pflegekinder (Betscher und Szylowicki 2016) ergänzt. Zwar sind nicht alle Gastfamilien, wie das Beispiel unten verdeutlicht, von der Kinder- und Jugendhilfe zusammengeführt worden, dennoch stellen solche Materialien Normierungen des sozialen Feldes dar, die hier exemplarisch aufgezeigt werden sollen.

\subsection{Differenzbearbeitungen im familialen Kontext}

Um die Besonderheiten der Gastfamilie herauszuarbeiten, gilt es hier also zunächst zu rekonstruieren, wie mit Differenzen und dementsprechend auch mit Moral und 
Moralisierung im Kontext von Familie idealtypisch umgegangen wird. Die folgende Darstellung dient hier also als Vergleichshorizont, an dem entlang mittels Kontrastierung Spezifika der Gastfamilie pointierter herausgearbeitet werden können.

Aus entwicklungspsychologischer und soziologischer Perspektive (Mead 1934 und Berger und Luckmann 2000 [1966] seien hier nur exemplarisch genannt) wird die Familie demnach zuallererst als Erziehungs- und Sozialisationsort für das heranwachsende, sich bildende Individuum angesehen. Oder wie Müller $(2007,150)$ schreibt: »Nirgendwo sonst in der Gesellschaft findet man ein gleichermaßen dichtes, komplexes und dauerhaft durch personale Generationsbeziehungen vermitteltes Feld zur Einführung der Nachkommenschaft in Kultur und Gesellschaft als in der Familie.«

Ohne auf die entwicklungspsychologischen Aspekte hier genau eingehen zu können, scheint es fruchtbar zu sein, zu betrachten, unter welchen Bedingungen die Familie das Individuum sozialisiert, erzieht und somit auch mehr oder weniger explizit moralisiert. Also das Individuum mit den Normen und Werten der Gesellschaft über Praktiken der moralischen Sanktionierung vertraut macht. ${ }^{4}$ So zeichnet die Familie als moralischen (Handlungs-)Raum aus, dass Müller $(2007,151)$ in Anlehnung an Claessens (1972) davon ausgeht, dass die »Ausbildung einer kulturellen Basispersönlichkeit auf der Grundlage emotional und sozial stabiler Primärbeziehungen, verlässlicher Erwartungsstrukturen (>sozialer Optimismus $<$ ) und emotional verankerter Werthaltungen « erfolgt.

Für die weiteren Ausführungen des Beitrags ist es zudem relevant, dass Müller (2007, 154f.) gleichzeitig von Differenzen ausgeht, die im familiären Raum in ihrer jeweiligen Eigendynamik auftreten. Differenzen liegen dabei nicht nur zwischen den Generationen, sondern auch zwischen den Eltern vor und können sich mit Blick auf unterschiedliche traditionelle oder moderne Wertorientierungen in Bezug auf Aufgabenverteilung und zu erreichende Bildungsaspirationen äußern (vgl. auch Sieben und Mey 2016). Auch wenn die Werte der Familienmitglieder miteinander in Konkurrenz treten mögen, bleiben bei einer Familie den oben angeführten Definitionen von Müller und Claessens zufolge die Interaktionen und die Beziehungen der Familienmitglieder zueinander trotzdem bestehen. Durch die gemeinsame Bearbeitung dieser Differenzen entwickle die Familie ihren Eigensinn und werde so zu einem Bildungsort im ko-konstitutiven Sinne für alle Familienmitglieder, der sich unter anderem in der »paradoxe[n] Dynamik von Kontinuität und Differenz « formiert (Müller 2007, 153f.). Familiale Räume zeichnen sich also auch dadurch aus, dass sich Eltern und Kinder wechselseitig moralisieren und damit normieren und sozialisieren. Dadurch werden nicht nur die Heranwachsenden als Subjekte im familialen Raum konstituiert, sondern auch die Erwachsenen als Subjekte umformiert. Insbesondere sobald die Kinder eigene Wert- und Normvorstellungen entwickeln, handeln Familien zudem einen sich immer wieder verändernden Werterahmen miteinander aus. 


\subsection{Differenzbearbeitungen zwischen Gast und Familie}

In Abgrenzung zum Familienbegriff wird hier nun auf den Begriff des »Gasts « und seine möglichen Bedeutungen für die Beziehungsgestaltung und Moralisierung in zweierlei Hinsicht eingegangen. Zum einen in seiner Bedeutung für die Erziehung und Sozialisation und somit auch Subjektumbildung des Unbegleiteten, der als Gast zur Familie hinzustößt. ${ }^{5}$ Und zum anderen mit Blick auf die Subjektumbildung aller Familienmitglieder, die sich in einem neuformierenden familialen Raum als soziales Gebilde, welches einen Eigensinn entwickelt, anders konstituieren müssen. Auch hier geht es also um eine Subjektumformierung in beide Richtungen. Als Besonderheit wird hier herausgearbeitet, dass die Gastfamilie im Vergleich zur »klassischen Familie « ein neues, temporäres und dadurch mitunter auch prekäres Zusammenleben darstellt und der Unbegleitete als (kulturell) Fremder hinzukommt.

Eine Besonderheit ist, dass der Begriff »Gast « auf ein temporäres Verweilen in der Familie hindeutet und somit auch die Neuordnung beeinflusst. Zum einen kann dies stabilisieren, da Differenzen temporär toleriert oder sogar gewünscht werden können, was dazu führen kann, dass sich die Beteiligten nicht so sehr aneinander anpassen müssen. Zum anderen ist der Gaststatus kein gesicherter Status in der Familie und macht deutlich, dass die Beziehung bei Nicht-Passung auch einfach beendet werden kann. Dies steht im Gegensatz zum Familienbegriff, bei dem von einer weitgehend unhinterfragten, lebenslangen Zugehörigkeit ausgegangen wird, die die Bearbeitung von Differenzen und die Entwicklung eines Eigensinns erst ermöglicht. Gleichzeitig erschwert die prekäre Zugehörigkeit des Unbegleiteten, dass die Familien der von institutioneller Seite an sie herangetragenen Zielvorstellung gerecht werden können, Schutz und Geborgenheit zu vermitteln (Betscher und Szylowicki 2016). Die oben genannten Charakteristika der »emotional und sozial stabile[n] Primärbeziehungen « sowie der Vermittlung »verlässlicher Erwartungsstrukturen « (Müller 2007, 151 in Anlehnung an Claessens 1972) sind zudem auch aufgrund der widersprüchlichen Anforderungen an die Anpassungs- und Integrationsleistung des Gasts im Gastfamiliensetting nicht gegeben. Die Subjektpositionierungen als Gast auf der einen und als Familienmitglied auf der anderen Seite wirken sich darüber hinaus auf die Spielräume der Moralisierung in diesem Handlungsraum aus und werden im Folgenden genauer vorgestellt.

Wird der Unbegleitete als temporärer, kulturell fremder Gast angesehen, so wird von ihm nicht erwartet, sich anzupassen. Da der Unbegleitete oft erst in der späten Adoleszenz in die Familie kommt, ${ }^{6}$ hat er seine Primärsozialisation schon erhalten. Gleichzeitig wird mitgedacht, dass der Unbegleitete bald schon auf eigenen Beinen stehen und nicht vom Kindes- bis zum Erwachsenenalter in der Familie bleiben muss. Dies anerkennend findet sich in Broschüren für Gasteltern zum Beispiel der Leitsatz »Begleiten statt erziehen « und »Ein gutes Ankommen, aber auch ein gutes $>$ Gehen $<[. .$.$] ermöglichen «$ 
(Becker 2016). In dieser Perspektive steckt eine hohe Wertschätzung der Primärsozialisation des Unbegleiteten. Dass der Gast in einigen Aspekten möglicherweise fremd ist, wird respektiert, da er nur eine Weile bleibt und sein eigenes Leben führen soll.

Gleichzeitig ist der Unbegleitete jedoch auch ein Gast in einem Familiensetting, welches für sich bestimmte Strukturen, Erwartungen und Zielvorstellungen entwickelt hat und mit bestimmten Werten und Normen bereits vor dem Zuzug des Jugendlichen Bestand hatte. Damit gehen gewisse implizite und explizite Erwartungen an den neuzugezogenen Unbegleiteten einher. Auch von einem Gast erwartet man, dass er sich an bestimmte Spielregeln im Haus hält, die von den GastgeberInnen festgelegt werden und ein gutes Zusammenleben ermöglichen. Bei Gastfamilien für unbegleitete Geflüchtete kommt hinzu, dass mit der Unterbringung in einem familialen Setting auch die Erwartungen der intergenerationalen und interkulturellen Kulturvermittlung an die Gastfamilien herangetragen werden. So wird als Vorteil einer Gastfamilie angesehen, dass sie zu einer individuellen und bedürfnisorientierten gesamtgesellschaftlichen Integration des Unbegleiteten beiträgt (Betscher und Szylowicki 2016, 5). Hier wird die Gastfamilie also als Sozialisations- und Integrationsinstanz adressiert, die die Perspektive eines längeren Aufenthalts im Ankunftsland, welche über den Gaststatus hinausgeht, mit sich bringt. Dementsprechend wird hier auch eine moralische Sozialisation des Unbegleiteten mit Blick auf die Vermittlung von Werten, Normen und Regeln und ein damit einhergehendes Sanktionieren von richtigen und falschen Verhaltensweisen nahegelegt.

Zugleich wurde jedoch bereits verdeutlicht, dass auch Familien Orte der wechselseitigen Moralisierung und somit auch der Sozialisation und Subjektumformierung sind. Dass der unbegleitete Geflüchtete die Rolle des Gasts und des kulturell Fremden einnimmt, führt auch dazu, dass der Handlungsraum von den Akteuren als interkultureller Handlungsraum gedeutet und verhandelt wird. Aus kulturpsychologischer Perspektive ist hier der Umgang mit dem Fremden und auch das Aushalten von Differenz bis mitunter sogar zum Entzug des Selbst- und vor allem des Fremdverstehens betrachtenswert (Boesch 1999; Freud 1919h; Krappmann 2016; Straub et al. 2007).

\subsection{Die Konstitution als Gastfamilie über (Alltags-)Moralisierungen}

Die vorgestellten Normierungen spiegeln sich auch im Interaktionsfeld selbst wider und legen dort bestimmte Subjektpositionierungen, Handlungspraktiken und Zielvorstellungen nahe. Es sollte deutlich geworden sein, dass Gastfamilie zwischen der Erwartung der Behandlung als kulturell Anderer - schützenswerter Gast - und der kompletten Anpassung als Familienmitglied ausgehandelt werden muss. Die kulturelle Fremdheit des jeweils Anderen sowie der unterschiedliche biografische Hintergrund führen dabei mitunter zu unterschiedlichen Bedürfnissen oder/und Verständnissen des 
Zusammenlebens (vgl. Gottschalk 2020). Diese Differenzen ergänzen die von Müller (2007) genannten Differenzlinien im familialen Raum. Dabei changieren Gastfamilien zwischen der unhinterfragten familialen Zugehörigkeit auf der einen Seite und der Fragilität eines neuen, temporären Handlungssettings auf der anderen.

Diese möglicherweise auch als Divergenzen erfahrenen unterschiedlichen Orientierungen werden von den Familienangehörigen gemeinsam bearbeitet. Unabhängig davon, inwiefern weitere unterschiedliche (kulturelle) Wertvorstellungen vorliegen, müssen Familien(-mitglieder) miteinander aushandeln, wie sie sich als Gastfamilie verstehen. Dabei steht die (Neu-)Konstitution des familialen Raums auch im engen Zusammenhang mit der (Neu-)Findung der eigenen Rolle in diesem Handlungssetting und geht mit einer Subjektumbildung aller Beteiligten einher. Dabei kommt es, wie in der Analyse im folgenden Kapitel exemplarisch gezeigt wird, immer wieder zu Irritations- und Aushandlungsmomenten in der sozialen Interaktion sowie in den Selbst- und Weltverhältnissen der einzelnen Individuen.

\section{Fallbeispiel Birgit:7 Zur situativen Aushandlungs- und Beziehungspraxis des Moralisierens im Alltag einer Gastfamilie}

Im Rahmen meiner Promotion, die dem vorliegenden Artikel zugrunde liegt, habe ich narrative Einzel- und Paarinterviews nach Schütze (vgl. 1983) mit problemzentriertem Fokus (Witzel 2000 [1989]) sowie Familiengespräche mit Gasteltern, Gastgeschwistern und Unbegleiteten geführt. Die Interviews wurden zwischen Herbst 2016 und Sommer 2019 in Deutschland, konkret in Nordrhein-Westfalen und in Bayern, durchgeführt. Das Sampling umfasst sowohl Familien, die durch die Kinder- und Jugendhilfe zusammengeführt wurden, als auch Familien, die über ein ehrenamtliches Engagement zueinander gefunden haben. Mithilfe einer interpretativen Vorgehensweise (Rosenthal 2014) soll rekonstruiert werden, welche Bedeutungen sich in den Subjektpositionierungen sowie Alltags- und Beziehungspraktiken der Gastfamilie wiederfinden lassen.

Für das Fallbeispiel wird eine längere Sequenz aus einem Interview mit der Gastmutter Birgit vorgestellt, die Hanad gemeinsam mit anderen ehrenamtlich begleitet. Insgesamt wurden zwei Interviews mit Birgit geführt. ${ }^{8}$ Die unten stehende Sequenz, die später analysiert wird, ist ein Ausschnitt aus dem ersten Interview mit Birgit. Besonders interessant ist das Beziehungssetting. So schreibt sie in ihrer ersten E-Mail:

»Ich betreue - in etwas ungewöhnlicher Form, daher evtl. interessant für Ihr Projekt? gemeinsam mit meinem Lebensgefährten, meiner Tochter sowie einem befreundeten Paar, das ganz in der Nähe wohnt, einen inzwischen 19-jährigen Flüchtling aus Somalia. Er wohnt unter der Woche in einer Gemeinschaftsunterkunft in der Nähe von [Stadt in 
Nordrhein-Westfalen], ist aber immer an den Wochenenden von freitags bis sonntags bei uns beziehungsweise bei der anderen $>$ Ersatzfamilie $<$. Wir $>$ teilen $<$ die Betreuung, weil wir alle vier beruflich sehr engagiert sind und wir uns sonst übernommen hätten.«

Eine Besonderheit des Fallbeispiels ist, dass mit den verschiedenen Unterkunftsorten viele Akteure an der Begleitung und Betreuung von Hanad beteiligt sind. Da Birgit im Verlauf des Interviews die gemeinsame Betreuung von Hanad zusammen mit dem anderen Paar als »erweiterte Familie « bezeichnet, wird das Fallbeispiel hier auch als eine Form von gelebter Gastfamilie verstanden. Durch die verschiedenen Unterkunftssettings werden jedoch unterschiedliche Norm- und Wertvorstellungen an Hanad und dadurch auch an Birgit herangetragen. Bei anderen Unbegleiteten mögen solche Konflikte ebenfalls auftauchen, sodass Birgit und Hanad hier nur beispielhaft angeführt werden. Auch wenn sie nicht wie Hanad mehrere Unterkunftsorte haben, gibt es mitunter zusätzlich Eltern oder Verwandte im Herkunftsland oder an einem anderen Ort, die an die Jugendlichen Erwartungen und Werthaltungen herantragen.

Die Schilderungen von Birgit wurden ausgesucht, da sie eine Aushandlungssituation beschreiben, in der Moralisierung sehr explizit wird. Durch die gemeinsame Bearbeitung der Moralisierung mit verschiedenen Akteuren wird nicht nur der gemeinsame Orientierungsrahmen ausgehandelt, sondern es kommt durch begleitende Reflexionen auch zu einer Neuorientierung von Birgits Selbst- und Weltverständnis. Die Sequenzen zeigen auf, wie Birgit davon irritiert ist, dass für sie als selbstverständlich angenommene Vorstellungen von Hanad zunächst nicht erfüllt werden, dann von ihr zunächst als gegeben und unverrückbar kommuniziert und schließlich mit unterschiedlichen Akteuren explizit verhandelt werden. Darüber hinaus veranschaulichen sie, wie an Hanad adressierte moralische Forderungen zu weiteren Moralisierungen von Akteuren führen, die an Hanads Sozialisation beteiligt sind, um das Ideal auch in der Eigengruppe zu realisieren. Gleichzeitig geben die dargestellten Sequenzen aus dem Fallbeispiel Einblick in die Grenzen von Moralisierung in komplexen Beziehungssettings sowie in Handlungsund Deutungsstrategien, um Moralisierung zu entschärfen und somit ein Zusammenleben zu ermöglichen.

Die von Birgit dargestellte Aushandlungssituation spinnt sich über mehrere Sequenzen. In den folgenden Abschnitten wird der Ablauf der Sequenzen rekonstruiert. Dazu werden die meisten Sequenzen in Form von Zitaten dargelegt und interpretiert. Auslassungen werden kenntlich gemacht und zum Teil zusammengefasst. Beim Lesen mag dabei der Eindruck entstehen, dass die Autorin selbst Birgit für ihr moralisierendes Verhalten verurteilt. Dazu sei gesagt, dass dies selbstredend nicht Intention des Beitrags ist. Letztendlich sagt es jedoch etwas über die diskursive Verortung des Forschungsfeldes aus und wie moralisch aufgeladene Diskurse, wie der Umgang mit Vielfalt, auch in Forschungsarbeiten verhandelt werden. Eine ausführliche Reflexion der Rolle und ge- 
wiss auch Verantwortung als Forscherin in solch einem Forschungsfeld wird an anderer Stelle vorgenommen.

\subsection{Einführung des Putzens als relevante Tätigkeit für (Alltags-)Moralisierungen}

In den im Folgenden dargestellten Moralisierungen geht es um das Putzen, das Birgit als Ausdruck einer Wertvorstellung einführt. In der entsprechenden Interviewsequenz berichtet Birgit zunächst, dass sie sich, um Vorbehalten entgegenzuwirken, insbesondere bei KollegInnen bemüht, viele positive Dinge über Hanad zu erzählen. Als Beispiel für solch eine positive Sache erzählt sie mir davon, dass Hanad in der Unterkunft, in der er unter der Woche wohnt, für eine erkrankte afrikanische Frau mit Kind kocht und sich zeitweise um das Kind kümmert. Die Frau wird später noch relevant werden und von Birgit als Helen vorgestellt.

Interessant ist, dass sie im Anschluss an diese Sequenz folgendermaßen fortführt:

»Also ich weiß nicht, ob wir ob wir auf so etwas noch zu sprechen kommen, es gibt auch Dinge, die nicht gut laufen, ne?//I: mhm//Also das sind so kulturelle Unterschiede (.). PUTZEN ist das Stichwort (.) und deswegen denke ich, das tut ihm gut, dass er sich auch mal da kümmern muss und dass er kochen muss. Und das tut er auch. (.).«

Putzen wird hier von ihr als etwas eingeführt, das exemplarisch für Dinge steht, die nicht gut laufen. Als Begründung werden hier kulturelle Unterschiede angeführt. Dass er sich »auch mal da kümmern muss und dass er kochen muss « wird hingegen als etwas, das ihm guttut, gerahmt. In Zusammenschau mit den nachfolgenden Sequenzen und den unten stehenden Interpretationen lässt sich dieser Ausschnitt so betrachten, dass Birgit solche Erfahrungen heranzieht, um für sich zu legitimieren, dass er, auch wenn er nicht putzt, andere als weiblich konnotierte Tätigkeiten übernimmt und er somit auch mit seinen eigenen Taten verdeutlicht, dass er die Gleichberechtigung von Mann und Frau ernst nimmt.

\subsection{Moralisierungen eines Individuums als Teil eines kulturell unterschiedlichen Kollektivs}

Das Putzen wird von mir mit einer immanenten Nachfrage wieder aufgegriffen, in der ich frage, wo » bei der Integration oder jetzt mit dem Putzen hatten Sie eben angedeutet, wo [...] da die Herausforderungen bei Ihnen [sind] «. Daraufhin führt sie kulturelle 
Unterschiede als ein »wirklich ein wirklich krasses Problem « ein, wobei die Wiederholung und das Stottern hier schon andeuten, dass sie die Thematik auch emotional sehr beschäftigt. Dazu verweist sie auf Erfahrungen, die sie, aber auch der Hausmeister und ehrenamtlich in der Unterkunft arbeitende Studierende, mit männlichen Geflüchteten aus der Unterkunft von Hanad gemacht haben:

»Also//I: mhm//dass die Gleichberechtigung von Mann und Frau eben bei manchen (holt tief Luft). Das ist jetzt bei unserem nicht der Fall. Also das akzeptiert er glaube ich wirklich. Aber also, ich sag jetzt nur mal das, was ich wirklich individuell jetzt wirklich berichten kann, ist ehm KRASSER kultureller Unterschied eh was zum Beispiel ehm Tätigkeiten angeht. Also die Männer und Frauen verüben oder verrichten.«

In dem hier präsentierten Ausschnitt wird Hanad als Teil eines Kollektivs von männlichen Geflüchteten eingeführt, von dem Birgit ausgeht, dass sich manche von ihnen mit der Gleichberechtigung von Mann und Frau schwertun. Hanad wird dabei zunächst mit den Worten »glaube ich wirklich « zumindest als erhofftes Ausnahmeexemplar dargestellt. Hier wird sich auf die Ausgestaltung von Geschlechterverhältnissen und Genderrollen in Deutschland und eine, aus ihrer Sicht, generalisierte Vorstellung vom » richtigen « Verhalten in Deutschland bezogen. Der Verweis von Birgit darauf, dass die Beobachtung von den Studierenden und dem Hausmeister geteilt werden, lässt sich dabei als Versuch verstehen, die Glaubwürdigkeit ihrer Perspektive als nicht rein subjektive Meinung zu bezeugen. Es zeigt sich zudem, dass Wertvorstellungen als grundsätzlich geteilt wahrgenommen werden können, es aber zu einer Diskrepanz kommen kann, wenn diese nicht auch handlungspraktisch umgesetzt werden oder zunächst nicht so ausgeführt werden, wie Birgit sich das wünscht. Dass Birgit diese Situation im Interview so ausführlich darlegt, zeigt zudem, dass sie diese vermeintliche Diskrepanz beschäftigt und trotz der im Folgenden noch weiter ausgeführten Aushandlungsprozesse für sich (noch) nicht als gelöst anzusehen scheint.

\subsection{Explikation vermeintlich deutscher Vorstellungen egalitärer Geschlechterbeziehungen am Beispiel putzender Ersatzväter}

Die folgende Sequenz gibt Einblick darin, in welcher kommunikativen Form und Situation die Moral in der Alltagswelt zwischen Birgit und Hanad relevant wurde und in Erscheinung trat.

»Also putzen ist für ihn, das ist No Go. Also ich hab am Anfang [der Zeit, als er von der Familie begleitet wurde] ein Telefonanruf von ihm bekommen da hat er fast geheult 
und hat gesagt: >THEY HAVE CRAZY RULES.< Und ich hab immer gedacht, er meint die Schule//I: $\mathrm{mhm} / /$. Bis ich mal verstanden hab, dass er meinte, dass er putzen soll//I: $\mathrm{mh} / /$ in diesem Haus, in dem er jetzt wohnt. [...] Und dann hab ich ihm natürlich erst einmal einen Vortrag gehalten am Telefon (lacht), dass er selbstverständlich putzen muss, nach einem Putzplan. Wer das denn sonst putzen soll? Und er war natürlich der Meinung die Frauen mü/müssen das putzen $/ / \mathrm{mhm} / /$, die in dem Haus wohnen. Und dann hab ich gesagt: >Nein, so ist das nicht. Du bist jetzt in Deutschland. Und hier machen das Männer und Frauen.< Und hab ihm erklärt, dass hier mein Lebensgefährte und der andere Ersatzvater, dass die auch alle putzen und dass er das doch auch sehen würde, wenn er hier ist.«

Birgit verweist hier auf vermeintlich universelle Vorstellungen der Ausgestaltung sozialen Miteinanders im deutschen Kulturraum. Besonders interessant ist die Bemerkung »Nein, so ist das nicht. [...] Und hier machen das Männer und Frauen «. Putzen wird hier zum Symbol für Gleichberechtigung. Birgit verweist dabei auf Vorstellungen von egalitären Geschlechterrollen, die vermeintlich im eigenen erweiterten Familienkreis auch so von den putzenden Männern vorgelebt werden. Dabei muss dieser Umstand von Birgit explizit gemacht werden, denn dass die Ersatzväter das ja auch implizit so vorleben würden, scheint nicht handlungsmotivierend auf Hanad zu wirken - was im Hinblick auf den weiteren Verlauf der von Birgit erzählten und im Folgenden von mir weiter analysierten Geschichte auch nicht verwunderlich ist, denn die Männer der beiden Gastfamilien putzen am Wochenende nicht.

Die Wortwahl »Und dann hab ich ihm natürlich erstmal einen Vortrag gehalten am Telefon (lacht) « sowie ihr Lachen zeigen, dass es für sie selbstverständlich und gleichzeitig vielleicht auch abstrus ist, diese Handlungspraxis erklären zu müssen. Während es für Hanad »Crazy Rules « sind, die nicht in sein Selbst- und Weltverhältnis passen, versteht Birgit zunächst erst gar nicht, worauf er sich mit seiner Aussage bezieht. Gleichzeitig zeigt das Wort »Vortrag «, dass es sich hier vielmehr um einen Monolog, wenn nicht sogar eine Standpauke gehandelt hat und nicht um einen Dialog oder Austausch über unterschiedliche Vorstellungen der Ausgestaltung von Geschlechterbeziehungen. Seine Meinung zählt hier nicht wirklich, sondern wird im Rahmen des Interviews vermutlich nur angeführt, um zwei konträre Weltsichten gegenüberzustellen. Birgits Ausführungen lässt sich derweil entnehmen, dass sie eine essenzialistische Sichtweise auf das oben von ihr bereits angeführte Kollektiv männlicher, wie sich im Interview immer wieder zeigt, insbesondere muslimischer Geflüchteter hat.

Besonders interessant ist dabei, dass sich ihre Handlungsaufforderungen nicht nur auf die familialen Räume der »erweiterten Familie « beschränken, sondern sich auch und sogar zunächst nur auf die neu zugeteilten Putzregeln in der Gemeinschaftsunterkunft beziehen. Dass Gasteltern auch auf andere Bereiche des Lebens des Unbegleiteten Einfluss zu nehmen versuchen, ist nicht ungewöhnlich, stellt hier aber aufgrund der 
unterschiedlichen Wohn- und Begleitsettings eine besondere Form dar. Bemerkenswert ist, dass Birgit auch die normative Deutungshoheit über Hanads Leben und Verhalten in der Unterkunft unter der Woche haben möchte und die soziale Aushandlung der Beziehungsgestaltung somit über den Sozialraum der Gastfamilie hinausgeht.

\title{
2.4 Zur Herstellung des Ideals putzender Männer im eigenen Kreis
}

Darüber hinaus berührt das Telefonat zwischen Birgit und Hanad auch das Zusammenleben in den familiären Räumen selbst. Denn Birgit macht in der oben zitierten Sequenz bei Hanad explizit, dass die Ersatzväter in den Haushalten der befreundeten Paare auch putzen.

\begin{abstract}
»(.) Ab sofort hab ich natürlich dann nach diesem Telefonat hab ich natürlich alle Männer verdonnert, also jetzt. Die putzen sowieso alle, das ist gar keine Frage. Ich hab dann gesagt: Bitte jetzt noch OFFENSICHTLICHER und sozusagen möglichst auch dann, wenn er dabei ist und das SIEHT. Also wir sind ja jetzt nicht so//I: $\mathrm{mhm} / /$, dass wir immer dann putzen, wenn er gerade hier ist, sondern wir versuchen das ja auch ein bisschen so zu legen, dass er ehm, also dass er es hier schön hat am Wochenende//I: $\mathrm{mhm} / /$ und wir jetzt nicht immer nur putzen. Das haben wir dann ein bisschen umgestellt. Also wir haben jetzt seitdem es diesen Konflikt gab (holt tief Luft) ehm verstärkt also ne zeitlang darauf geachtet, also dass die Männer sich da wirklich auch mehr//I: mhm//mit diesen Dingen zeigen. (Holt tief Luft) $\ll$
\end{abstract}

Auffällig ist, dass der von Birgit angenommene Normalzustand erst hergestellt und inszeniert werden muss. Es wird also im Goffman'schen (1991 [1959]) Sinne ein pädagogisches, auf Veränderung abzielendes Theaterstück auf der Vorderbühne präsentiert. Hanad ist dabei nicht Teil der Inszenierung, sondern nur Zuschauer beziehungsweise Adressat. Dies wird von Birgit damit begründet, dass das Wochenende keine normale Situation, sondern einen Ausnahmezustand darstelle, in der man Zeit mit ihm verbringe wolle. Hanad wird in dieser Situation als Gast und nicht als selbstverständliches Familienmitglied positioniert. Auch wenn die Argumentation nachvollziehbar ist und viel über den besonderen Status von Hanad in den Familien sagt, werden die von Birgit proklamierten Normen und Werte dadurch dennoch auch ein Stück weit als Idealisierung der eigenen Gruppe entlarvt.

Der Idealzustand von Birgit wird, um für ihn als Vorbild dienen zu können, erst durch ihre Anweisung hergestellt. Jemanden zu etwas »verdonnern « lässt sich dabei als machtvoller moralischer Akt und als Form der Moralisierung der anderen Gasteltern verstehen. Nicht nur die oben angeführten gesellschaftlichen und institutionellen 
Normierungen wirken also moralisierend, sondern vermeintliche Ideale werden auch in der Alltagswelt aufgegriffen und reproduziert. Gleichzeitig ist solch eine Selbst- und Fremdmoralisierung unter den Gasteltern und innerhalb der Gastfamilie jedoch auch nötig. Denn wenn die Zielvorstellung ist, dass Hanad sich wie ein »guter Deutscher « verhält, dann müssen ErzieherInnen und BegleiterInnen das in der Tat auch selbst so vorleben. Am Kontext Gastfamilie mag dabei besonders sein, dass Gasteltern durch die oben exemplarisch herausgearbeitete Rahmung des Beziehungssettings mögliche ungewollte und fremde Charaktereigenschaften, Einstellungen und Verhaltensweisen erwarten, die sie dann auf vermeintliche und möglicherweise auch tatsächlich wirksame kulturelle Unterschiede zurückführen können.

Was hier in dieser Situation dennoch zunächst nicht passiert, oder zumindest nicht explizit gemacht wird, ist, dass das eigene Ideal selbstkritisch infrage gestellt wird. Birgit deutet die Situation rückblickend als Konflikt, der dazu geführt hat, dass sie ihn belehrt und sie ihr Verhalten »alle ein bisschen umgestellt haben «. Die Auseinandersetzung wird also über Verhaltensänderungen reguliert und nicht über eine Reflexion und Infragestellung der Wertvorstellungen. Dass sie ihre Unterweisung, die das Zusammenleben der Unterkunft berührt, als »Konflikt « deutet, ist besonders interessant. Auch wenn der Konflikt nicht durch einen irritierenden Vorfall im eigenen Haushalt ausgelöst wurde, berührt es Birgits Erwartungen an Hanad und stimmt nicht mit ihrem Selbst- und Weltverhältnis überein, sodass dieser sich anbahnende Konflikt in der Unterkunft vor allem zu einem inneren Konflikt bei Birgit führt. Hinzu kommt, dass im weiteren Verlauf ihrer Narration deutlich wird, dass Hanad bis zum Zeitpunkt des hier zitierten Interviews in der Gastfamilie nahezu gar nicht geputzt hat und Birgit hier mit ihrer praktischen Durchsetzungskraft ihrer Zielvorstellungen hadert. Birgit überdenkt in diesem Zuge also ihre eigenen Erziehungspraktiken und möchte sie anpassen. Im Anschluss an diese Sequenz berichtet sie daher, dass sie die Konfliktsituation als Anlass genutzt habe, ihm öfter am Wochenende einen Staubsauger in die Hand zu drücken und dass es ein weiteres Ziel von ihr ist, ihn öfter das Bad putzen zu lassen.

\subsection{Vermeintlicher Erfolg des »Erziehungseffekts»}

In dem Telefonat, in dem sie ihm einen »Vortrag « über putzende Männer in Deutschland gehalten hat, untermauert Birgit ihre Moralisierungen zudem folgendermaßen:

»Also das kann ich noch abrunden durch Folgendes. Ehm ich hab ihm das dann erklärt damals bei dem Telefonat, das war im Februar. (.) Dass er putzen muss und dass er sich Handschuhe kaufen soll. Diese gelben Handschuhe bei Rossmann. Das hat er auch verstanden. (.)//I: mhm//Also das war alles auf Englisch. Und dann hat er immer gesagt: >Ja, 
I know what you mean. $<$ Weil er diese gelben Handschuhe hier bei mir bereits gesehen hatte//I: mhm//. Also wenn er in der Küche neben mir steht und mit mir quatscht, mach ich ja oft irgendwas sauber und dann hab ich auch manchmal diese Handschuhe an. Also er wusste ganz genau, was ich meine. (.) Und dann hab ich gesagt, er soll sich die kaufen und soll bitte mit diesen Handschuhen putzen. Dann wär das nicht ganz so schlimm, wenn man [das] Klo putzt. Ich würd das auch nicht gerne machen. (holt tief Luft) Und dann hat er gesagt: Ja, das will er machen (.) Und eine Woche später hab ich ihn gefragt: >Wie hat es geklappt mit dem Putzen?<. Und dann hat er gesagt: Ja, die Helen, das ist diese junge Frau aus Eritrea, also die Mutter. Ehm die hätte ihm sehr geholfen und ihm alles gezeigt und so, weil er könnte das ja nicht und er wüsste ja gar nicht, wie man wischt und solche Dinge.«

Indem sie ihm von den gelben Handschuhen erzählt, die er schon bei ihr gesehen habe, verweist sie rückblickend auf konkrete Situationen, in denen sie als Vorbild agieren konnte. Gleichzeitig erklärt sie ihm, dass sie das Putzen auch nicht möge. Wichtig für den weiteren Verlauf der Geschichte ist hier, dass sie immer wieder betont, dass er ihre Ausführungen verstanden habe und dies letztendlich auch zum Erfolg geführt habe. Helen, die bereits zu Beginn der Geschichte benannt wurde, wird hier von ihr zunächst als Unterstützerin gesehen, die Hanad beim Putzen geholfen und somit Birgits Belehrungen unterstützt habe.

\subsection{Grenzen der Deutungshoheit und Moralisierung}

Dass Birgit den vorherigen Absatz mit den Worten »Also das kann ich noch abrunden durch Folgendes « als erzählerisches Element einleitet, deutet jedoch bereits an, dass eine Wende ansteht und etwas Überraschendes oder Spannendes folgt:

»So und eine weitere Woche später kommt er an und sagt eh freudestrahlend kam er hier an. Ich mach die Tür auf und dann sagt er: $>$ I don't clean anymore. $</ / \mathrm{I}$ : hmm//Und dann hab ich gesagt: >Hä, was soll das heißen, wieso putzt du nicht mehr?< (Holt tief Luft) NEIN, die Frauen in dem Haus wollen das nicht und die haben ihm das verboten, haben ihm alles weggenommen und haben gesagt: sie putzen. Das wär für sie unvorstellbar. Das sind ja Afrikanerinnen auch//I: $\mathrm{mhm} / /($.), dass sie sozusagen Toiletten und so weiter benutzten und dass die von den Männern geputzt werden. Und kurz darauf war ja dann die Geburtstagsfeier, wo ich dann in [Ort, in dem Hanad untergebracht ist] war. Und dann hab ich mir diese Helen natürlich sofort vorgeknöpft und hab also dann versucht, auf Englisch ihr klarzumachen, dass sie meine ganze Erziehung untergräbt und dass das//Interviewerin lacht//so nicht geht. Ehm sie hat aber sehr charmant reagiert und ehm das Ende vom Lied ist. Ich kann ja nicht, ich kann sozusagen nicht jetzt dieser ganzen Wohnge- 
meinschaft jetzt//I: $\mathrm{mhm} / /$ in diesem Haus, meine Vorstellungen aufoktroyieren, das geht einfach nicht. (.) Wenn die sich intern jetzt damit wohler fühlen und sich darauf einigen: Die Frauen möchten nicht, dass die Männer putzen, sondern sie möchten selber putzen und die Frauen/die Männer finden das sowieso super, dass sie nicht putzen müssen.«

Interessant ist, dass Birgit auch versucht, die bereits zwei Mal eingeführte Frau aus Eritrea, Helen, zu moralisieren. Ähnlich wie »verdonnern« ist auch »vorknöpfen « ein Ausdruck, der auf eine sehr stark explizit gemachte Moralisierung hinweist. Der Ausschnitt lässt sich so deuten, dass Birgit versucht, ihre Moralvorstellungen an weitere sozialisationsrelevante Akteure weiterzugeben und hier ihre Deutungsmacht ein- oder aus ihrer Sicht vielleicht auch zurückzufordern. Helen steht hier als afrikanische Frau und Mutter, wenn auch aus Eritrea und nicht aus Somalia, quasi sinnbildlich für eine Moralinstanz aus Hanads Heimatkultur, die die von Birgit vorgenommene Moralisierung zurückweist. Birgit empfindet das als »charmant « und kommt dadurch selbst zur Einsicht, dass sie ihre Vorstellungen der Hausgemeinschaft »aufoktroyieren « wollte. Damit hält Helen die Deutungshoheit und Handlungsmacht aufrecht, was auch von Birgit akzeptiert wird. Hier kommt Moralisierung nicht nur an ihre Grenzen, vielmehr moralisiert auch Helen Birgit durch ihre erfolgreiche Zurückweisung. Gleichzeitig gibt Helen Hanad dadurch die Legitimation, nicht putzen zu müssen.

\subsection{Einen Kompromiss finden}

Birgit versucht dennoch das aus ihrer Sicht Beste aus der Situation zu machen. Im Folgenden berichtet sie, wie sie versucht, einen Kompromiss zu finden, ihre Erziehungsziele als verwirklicht anzusehen und ihr positives Bild von Hanad aufrechtzuerhalten.

»(Holt tief Luft). Gut, dann ist das so. Das akzeptier ich jetzt auch, aber ich habe im Gegenzug ehm den Hanad dazu verdonnert und das hat er auch sofort akzeptiert und auch zurückgeschrieben: > This is a great idea. < Also das war ja alles damals noch auf Englisch. (.) Ehm ich hab ihm dann geschrieben, also er soll das einmal im Monat oder so nen Kuchen backen oder. Das kann er, das hat er inzwischen hier gelernt//I: mhm//. Er soll Kuchen backen oder er soll irgendwie Kekse einkaufen. Er soll irgendwas tun und das in die Küche stellen und eh, damit er auch sich beteiligt//I: mhm//und eine Gegenleistung bringt. Und dann hat er zuerst gesagt eh: >This is such a good idea.< oder so. Hat er zurückgeschrieben//I: mhm//. Und dann dachte ich: >Naja, ob er das jetzt auch macht?<//I: mhm//und ohne, dass ich ihn erinnert hätte, hat er dann tatsächlich ein, zwei Wochen später also ein Kuchen gebacken, abfotografiert, mir das Foto geschickt. (.) Und er erwähnt das ab und zu. Also//I: $\mathrm{mhm} / /$, dass er ehm, dass wieder vergessen hat und dass 
er jetzt doch wieder dran gedacht hat, irgendwas zu kaufen und hinzustellen. Also das ist jetzt nen Deal sagen wir mal (.), den find ich völlig akzeptabel. In anderen//I: $\mathrm{mhm} / /$ in deutschen Familien ist es ja auch nicht so, dass jetzt jeder Mann die/die Klos putzt//I: $\mathrm{mhm} / /$. Man kann immer irgendne, und er kann jetzt nicht mit ehm (.) Auto waschen oder reparieren sich (lacht) revanchieren. Also von mir aus dann eben mit Kuchen backen und was einkaufen//I: mhm//. Völlig in Ordnung. (Holt tief Luft). Aber man sieht, dass der Erziehungseffekt noch nicht richtig funktioniert hat, weil hier macht er wirklich null komma null, ne.//I: Mhm//. (.) Also da muss ich einfach noch ehm (.) es kommt einem komisch vor, dass man das bei einem 19-Jährigen eben jetzt oder damals 18-Jährigen erst so beginnt. Aber es ist eben so, ne.//I: mhm//. (.) Ja. Und in/in diesem kulturellen Bereich gibt es natürlich noch mehr Unterschiede.«

Birgit, die hier wieder das Wort »verdonnert « benutzt, verdeutlicht damit, dass sie ihre Anweisungen erneut als unumstößlich versteht. Das Kuchenbacken scheint sie als unabdingbare Gegenleistung zu verstehen. Somit wiegt sie das Nicht-Putzen mit anderen Handlungspraktiken auf, die vermeintlich weiblich konnotiert sind und mit denen sich das Untersagen des Putzens ausgleichen oder wie Birgit sagt » revanchieren « lässt. Dies lässt sich als Versuch verstehen, unter ungleichen Bedingungen einen Hauch von Egalität herzustellen. Wichtig scheint ihr hervorzuheben, dass er das Kuchenbacken kann und von ihr gelernt habe. Damit schließt sich der Kreis mit dem Beginn der Narration, wo es ihr - wir erinnern uns - wichtig war, positiv von Hanad erzählen zu können und zu erwähnen, dass er sich um die besagte Helen und ihr Kind kümmert und dort kocht.

Das Kuchenbacken versteht sie als »Deal«, als Kompromiss, den sie »völlig akzeptabel « findet. Zugleich erkennt sie hier, dass putzende Männer letztendlich doch nur eine Idealvorstellung sind, die auch in anderen deutschen Familien (nicht nur in der eigenen) nicht verwirklicht wird. Es findet also, nachdem sie ihre Vorstellungen zunächst recht unumstößlich formuliert hat, eine Relativierung vermeintlich deutscher Werte statt. Vielmehr kommt sie zu der Einsicht, dass Männer sich sonst oft mit männlich konnotierten Tätigkeiten revanchieren, mit denen sich Hanad aber nicht erkenntlich zeigen könne.

Dennoch bleibt Birgits Unzufriedenheit bis zum Ende dieser Geschichte bestehen, da sie davon berichtet, dass der »Erziehungseffekt « noch nicht richtig funktioniert habe. Das begründet sie zum einen damit, dass er bei den Familien zu Hause nicht putze. Zum anderen irritiert und ärgert es Birgit, dass sie das einem 18- oder 19-Jährigen überhaupt erklären muss. Der Verweis auf vermeintliche kulturelle Unterschiede schwächt diese Irritation ein wenig ab. Hier wirkt sich also der im vorangegangenen Kapitel herausgearbeitete » Gaststatus « des Unbegleiteten, der sich auch auf eine kulturell anders geprägte Primärsozialisation zurückführen lässt, konfliktentlastend auf die Beziehung zwischen Birgit und Hanad aus. 


\section{Zum Zusammenhang von Beziehungsgestaltung und Subjektbildung im Rahmen von Moral(-isierungen) - Abschließende Überlegungen}

Im Beitrag wurden Gastfamilien für unbegleitete Geflüchtete als Handlungsfeld vorgestellt, an das über Normierungen unterschiedliche Orientierungen der Beziehungsgestaltung und Spielräume der Moralisierung herangetragen werden. Die Gastfamilie wird so zu einem moralischen Raum, wobei die für die Familie als neu entstehender Sozialraum konkret gültigen Orientierungen der Moralisierung sowie Wertvorstellungen von den Gastfamilienmitgliedern in Alltagssituationen ausgehandelt werden. Gastfamilien sind dabei als ein sich im Wandel befindliches Zusammenleben zu verstehen, in welchem sich die Bildung des familialen Raumes und des Subjekts unter Bedingungen von Macht- und Verantwortungsdifferenzialen und emotionaler Nähe vollzieht.

Am Fallbeispiel von Birgit wurde gezeigt, wie sie auf vermeintlich generelle Vorstellungen der Ausgestaltung von Genderrollen in Deutschland zurückgreift, um dem Unbegleiteten Hanad das Putzen nahezulegen, das dieser als weiblich attribuierte Tätigkeit ablehnt. Allein durch ihre Wortwahl »verdonnern«, »vorknöpfen« und »Vortrag halten « macht sie Moralisierungen sehr explizit. An diesem Beziehungssetting ist besonders, dass es sich um ein komplexes soziales Feld handelt, in dem für Birgit vermeintlich selbstverständliche Normen und Werte mit vielen Akteuren ausgehandelt werden. Diese komplexe Beziehungsstruktur führt dazu, dass Moralisierungen in einem dynamischen Beziehungs- und somit auch Aushandlungssetting im Vordergrund stehen. Fragen zu Hanads Rolle im Rahmen der erweiterten Familie, ob vor ihm als »Gast « geputzt wird oder nicht und ob er als Familienmitglied sogar selbst putzen muss, werden so aufgeworfen und wollen behandelt werden.

\subsection{Von der Kulturalisierung des Fremden zum Bruch mit der Illusion der Einheit in Vielfalt}

Das Beispiel zeigt eindringlich, dass Moralisierungen genutzt werden können, um eine Eigengruppe zu kreieren. Hier wird das zunächst darüber vorgenommen, dass das Fremde, also auch die nicht nahestehenden Wertvorstellungen, kulturalisiert werden, um das eigene Selbst zu erhöhen und Wir-Ihr-Bilder zu erzeugen. Zum anderen verdeutlicht das Beispiel jedoch auch Grenzen der Moralisierung und Selbsterhöhung in einem komplexen Beziehungssetting.

Anhand von Birgits Ausführungen zeigt sich sehr gut, dass die erweiterte Familie rund um Hanad keinen isolierten Handlungsraum darstellt, sondern eng mit anderen Handlungsräumen verschränkt ist, durch die sich das Individuum bewegt. Exempla- 
risch lässt sich hier erkennen, dass in einer pluralen Gesellschaft auch generalisierte Vorstellungen vom richtigen oder falschen Verhalten, wie die Idee, dass in Deutschland auch alle Männer putzen, obsolet werden.

Das merkt auch Birgit, die Hanads Verhalten zunächst zu moralisieren versucht. An den Aushandlungen, die sie in der Folge mit den anderen Ersatzfamilienmitgliedern und Helen führt, lässt sie ihn hingegen nicht teilhaben. Mit Hanad führt sie unabhängig von den anderen Gespräche, die einen Aufklärungscharakter haben.

Besonders interessant ist am Fallbeispiel selbst, wie Helen die Moralisierung zurückweist und damit Birgit zum Nachdenken anregt. Birgit wird so auf die Grenzen ihres eigenen Handelns und die Begrenzung ihrer Moralvorstellungen zurückgeworfen. Sie führt dies auf interkulturelle Unterschiede zurück. Letztendlich muss Birgit jedoch auch den eigenen erweiterten Familien- und Freundeskreis moralisieren, um das Ideal der putzenden Männer herzustellen.

Die (Alltags-)Moralisierungen im Gastfamilienraum stehen hier Pars pro Toto für Aushandlungen, die auch in anderen Handlungs- und Beziehungssettings stattfinden. Moralische Inhalte und Moralisierungen sind dabei im Rahmen der Beziehungsaushandlung immer situativ und fluide. Die Vielfalt und die damit einhergehenden Differenzen müssen sowohl bei der (Neu-)Konstitution eines Handlungsraums wie der Gastfamilie als auch von den Subjekten ausgehalten und in das Familien- und das eigene Selbst- und Weltverhältnis integriert werden. Im Rahmen der (Neu-)Formierung einer Beziehung muss sich das Subjekt also immer wieder neu konstituieren und verorten. Aus den vorangegangenen Überlegungen lassen sich daher weitere Rückschlüsse für die Moral(-isierung) und die Bildung und Gestaltung von Beziehungsräumen sowie auf die Subjektbildung ziehen.

\subsection{Moral(-isierung) und die Bildung und Gestaltung von Beziehungsräumen}

Besonders herausforderungsreich scheint die Beziehungsaushandlung demnach, wenn die Beziehung nicht klar gerahmt ist und neben Differenzen in den Wertorientierungen auch die Beziehungsorientierung selbst nicht geklärt ist. Wenn in einer Gastfamilie uneindeutig ist, ob die neu hinzugekommene Person als Gast oder als Familienmitglied behandelt werden soll, ist möglicherweise unklar, inwiefern moralisierende Anpassungsforderungen an den Unbegleiteten gestellt werden können - im Fall Hanad also, ob er auch im Haushalt von Birgit putzen muss oder wie ein Gast am Wochenende behandelt wird.

Handlungsräume als Beziehungsräume bilden sich also in einem Spannungsverhältnis von Kontingenz und Wandel einerseits sowie Stabilität andererseits. Zum einen bietet Stabilität eine Chance für die Wertorientierung aller Beteiligten. Im Fall der 
Gastfamilie wird dies insbesondere als Gewinn für den Unbegleiteten gesehen. Zum anderen kann die Neuausrichtung mit einem Orientierungsverlust für alle Beteiligten einhergehen.

Das Beispiel zeigt zudem, dass Moralisierung ihre Grenzen hat und nicht immer erfolgreich ist, beispielsweise weil die moralische Deutungshoheit über einen bestimmten Bereich fehlt oder die andere Person sich nicht moralisieren lässt. Birgit muss, um weiterhin gut mit Hanad zusammenzuleben, ihre Moralisierungen relativieren. Zusammen mit Hanad findet sie einen Kompromiss, sodass in Birgits Augen unmoralisches Verhalten akzeptiert und die Beziehung aufrechterhalten werden kann. Solchen flexiblen, situativen Praktiken der Entschärfung von (Alltags-)Moralisierungen scheint insbesondere in fragilen Orten des Miteinanders eine wichtige psychosoziale und soziokulturelle Funktion zuzukommen. Empirisch interessant ist, wie solche Kompromisse aussehen und wie für alle an der Moralisierung beteiligten Akteure ein gutes Miteinander hergestellt werden kann. Alternativ könnten sich die Personen auch voneinander distanzieren, die Beziehung aufgeben oder umdeuten, wenn Moralvorstellungen vermeintlich auseinandergehen. Für die Gestaltung von Beziehungen und insbesondere von solchen in Gastfamilien, die sowohl erziehen beziehungsweise sozialisieren als auch integrieren, ist die Frage interessant, wie viel Fremdheit in solchen Settings ausgehalten wird und wie viel zumindest angenommene Übereinstimmung es im Zusammenleben braucht. Zudem dürfte deutlich geworden sein, dass Beziehungsgestaltung immer als wechselseitiges Aufeinanderzugehen zu verstehen ist, sodass auch moralische Vorstellungen einer einseitigen Integration des Unbegleiteten in die Familie und von Geflüchteten in die Gesellschaft durch die vorangegangenen Überlegungen fraglich sein sollten.

\subsection{Moral(-isierung) und Subjektumbildungen}

Auf der individuellen Ebene setzt das voraus, dass sich die beteiligten Personen dafür öffnen, die Stabilität ihrer eigenen Werte infrage zu stellen und sich selbst zu reflektieren. Nicht nur im Gastfamiliensetting, sondern auch in anderen Beziehungen mag mitunter im Zeitalter der Kontingenz viel dadurch gewonnen sein, die Ambiguität von differenten Wertvorstellungen und somit auch Handlungsorientierungen anzuerkennen und auszuhalten (Krappmann 2016).

Letztendlich kommt Birgit dazu, die vermeintliche Allgemeingültigkeit infrage zu stellen. Hier zeigt sich exemplarisch, was insbesondere als Lerneffekt interkultureller Begegnungen betrachtet wird (Straub et al. 2007): (Interkulturelle) Differenzerfahrungen können das eigene Selbst- und Weltverhältnis irritieren und zu moralischen Reflexionen anregen. Analog zu den oben vorgestellten Bildungsprozessen des Handlungsraums 
wird dadurch nicht nur die Orientierungsfunktion der eigenen Werte infrage gestellt, sondern es kann auch zu einer Horizonterweiterung und somit Subjektumbildung aller Gastfamilienmitglieder kommen.

\section{Anmerkungen}

1 Den HerausgeberInnen Anna Sieben und Paul Sebastian Ruppel sowie den Gutachterlnnen sei für die hilfreichen Anmerkungen zur Überarbeitung herzlich gedankt.

2 Die Begriffe »Gast-« und »Pflegefamilie« werden im Rahmen des Beitrags synonym verwendet. Auf den Begriff der Gastfamilie wird zurückgegriffen, da sich, wie unten gezeigt wird, spezifische Merkmale der Beziehungsform daran besonders gut veranschaulichen lassen.

3 Die hier genannten Namen sind Pseudonyme.

4 Interessant ist es auch, den Zusammenhang von Sozialisation im familiären Kontext und die Ausbildung von moralischem Denken, Gefühlen und Verhalten, also eines moralischen Selbst in den Blick zu nehmen.

5 Da 201691,7 Prozent der Unbegleiteten männlich gewesen sind (Tangermann und HoffmeyerZlotnik 2018, 19) und sich auch im Sample des diesem Beitrag zugrunde liegendem Promotionsvorhabens nur männliche Geflüchtete befinden, wird hier ausschließlich die männliche Form verwendet.

693 Prozent der Unbegleiteten, die im Jahr 2016 eingereist sind, waren zwischen 14 und 17 Jahren (Tangermann und Hoffmeyer-Zlotnik 2018, 18).

7 Ausschnitte des Transkripts wurden in einem Kolloquium mit Prof. Dr. Jörg Bergmann (»Warum lässt du immer deine Socken ... - Konstellationen, Formen und Praktiken der (in)direkten Moralisierung im Alltag«, Hans Kilian und Lotte Köhler Centrum, 23. Januar 2019, Ruhr-Universität Bochum) diskutiert. Ihm und den Teilnehmenden sei für hilfreiche Interpretationen und Anmerkungen herzlich gedankt.

8 In Gottschalk (2020) werden die Beziehungsdeutungen von Birgit und Hanad einander gegenübergestellt.

\section{Literatur}

Becker, Lilith. 2016. „Gastfamilie für minderjährige Geflüchtete gesucht. Wie nehme ich einen unbegleiteten minderjährigen Flüchtling (UMF) auf?«. evangelisch.de. Zugriff 13.04.2020. https://www.evangelisch.de/inhalte/138144/13-09-2016/wie-nehme-ich-einen -unbegleiteten-minderjaehrigen-fluechtling-auf-umf-auf.

Berger, Peter L. und Thomas Luckmann. 2000 [1966]. Die gesellschaftliche Konstruktion der Wirklichkeit. Eine Theorie der Wissenssoziologie. 17. Aufl. Frankfurt a. M.: Fischer Taschenbuch Verlag.

Bergmann, Jörg. 1999. »Alltagsmoral - Eine Erkundung". In Kursbuch 136: Schluss mit der Moral, hrsg. v. Karl Markus Michel, Ingrid Karsunke und Tilman Spengler, 105-20. Berlin: Rowohlt.

Betscher, Silke und Alexandra Szylowicki. 2016. Jugendliche Flüchtlinge in Gastfamilien. Eine erste Orientierung in einem großen gesellschaftlichen Feld. Hrsg. v. Kompetenz-Zentrum Pflegekinder e.V. Berlin. https://www.kompetenzzentrum-pflegekinder.de/workspace/uploads/ jugendliche-fluechtlinge-in-gastfamilien_1.pdf. Zugriff 01.11.2020.

Boesch, Ernst E. 1991. Symbolic Action Theory and Cultural Psychology. Berlin: Springer (Recent Research in Psychology). 
Boesch, Ernst E. 1996. »Das Eigene und das Fremde«. In Psychologie interkulturellen Handelns, hrsg. v. Alexander Thomas, 87-105. Göttingen, Bern, Toronto \& Seattle: Hogrefe.

Claessens, Dieter. 1972. Familie und Wertsystem. Eine Studie zur "zweiten, sozio-kulturellen Geburt» des Menschen und der Belastbarkeit der »Kernfamilie». Berlin: Duncker \& Humblot (Soziologische Abhandlungen, 4).

Freud, Sigmund. 1919h. Das Unheimliche. In Gesammelte Werke, Bd. XII, 227-68.

Goffman, Erving. 1991 [1959]. Wir alle spielen Theater. 7. Aufl. München: Piper.

Gottschalk, Ines. 2020. »Zwischen Familie und Engagement: ‘Gastfamilien als polyvalente Integrationsorte für unbegleitete Geflüchtete«. In Integration Geflüchteter in Deutschland, hrsg. v. Anna Mratschkowski, 153-183. Baden-Baden: Nomos.

Krappmann, Lothar. 2016. Soziologische Dimensionen der Identität. Strukturelle Bedingungen für die Teilnahme an Interaktionsprozessen. Zugl.: Berlin, Freie Univ., Diss., 1969. 12. Aufl. Stuttgart: Klett-Cotta.

Mead, George Herbert. 1934. Mind Self and Society from the Standpoint of a Social Behaviorist. Hrsg. v. Charles W. Morris. Chicago: University of Chicago.

Müller, Hans-Rüdiger. 2007. „Differenz und Differenzbearbeitung im familialen Erziehungsmilieu. Eine pädagogische Problemskizze«. Zeitschrift für Soziologie der Erziehung und Sozialisation 27 (2): 143-59.

Rosenthal, Gabriele. 2014. Interpretative Sozialforschung. Eine Einführung. 4 Aufl. Weinheim: Beltz Juventa.

Schütze, Fritz. 1983. »Biographieforschung und narratives Interview«. Neue Praxis 3: 283-93.

Sieben, Anna und Günter Mey. 2016. »Editorial«. Journal für Psychologie 24 (1): 1-9.

Straub, Jürgen. 1994. »ldentität und Sinnbildung. Ein Beitrag aus der Sicht einer handlungs- und erzähltheoretisch orientierten Sozialpsychologie«. ZiF Jahresbericht 1994/95.

Straub, Jürgen, Arne Weidemann und Doris Weidemann, Hrsg. 2007. Handbuch interkulturelle Kommunikation und Kompetenz. Grundbegriffe - Theorien - Anwendungsfelder. Stuttgart \& Weimar: Verlag J. B. Metzler.

Taylor, Charles. 2006 [1992]. Quellen des Selbst. Die Entstehung der neuzeitlichen Identität. 9. Aufl. Frankfurt a. M.: Suhrkamp.

Witzel, Andreas. 2000 [1989]. »Das problemzentrierte Interview«. Forum Qualitative Sozialforschung/ Forum: Qualitative Social Research 1 (1), Art. 22.

\section{Die Autorin}

Ines Gottschalk, M. A., Doktorandin am Lehrstuhl für Sozialtheorie und Sozialpsychologie von Prof. Dr. Jürgen Straub an der Ruhr-Universität Bochum sowie wissenschaftliche Mitarbeiterin im Projekt »Forschungsbezogenes Schreiben und öffentliches Präsentieren « des QPL-Projekts inSTUDIES. Forschungs- und Lehrschwerpunkte: Kulturpsychologie, Qualitative Forschung, Biografieforschung, Flucht- und Migrationsforschung, Figurationssoziologie, Engagementforschung, Öffentliche Soziologie, Forschendes Lernen, Digitalisierung und Schreiben.

Kontakt: Ines Gottschalk, Ruhr-Universität Bochum, inSTUDIES Projektbüro, Universitätsstraße 105, 1. OG, Raum 1.27, 44789 Bochum; E-Mail: ines.gottschalk@rub.de 